\title{
Intrinsic Complexity of RR and QT Intervals at the Cellular Level
}

\author{
Jiyeong Kim ${ }^{1}$, Ilya Potapov ${ }^{1}$, Disheet Shah ${ }^{2}$, Jukka Kuusela ${ }^{2}$, Katriina Aalto-Setälä ${ }^{2,3}$, Esa Räsänen ${ }^{1}$ \\ ${ }^{1}$ Tampere University of Technology, Tampere, Finland \\ ${ }^{2}$ BioMediTech, University of Tampere, Tampere, Finland \\ ${ }^{3}$ Heart Hospital, Tampere University Hospital, Tampere, Finland
}

\begin{abstract}
We characterize the complexity of interbeat intervals (IBIs) and field potential durations (FPDs) of humaninduced plurioptent stem cell-derived cardiomyocytes (hiPSC-CMs). The complexity is assessed using the multiscale entropy (MSE) method up to the scale of ten beats. The MSE profiles of healthy and diseased (long-QT syndrome) hiPSC-CMs show important differences, which demonstrate the usefulness of the method in the characterization of the cells and in the analysis of hereditary cardiac diseases. We show that the intrinsic complexity is possibly altered by the differentiation methods, as well as the age and mutations of the cells.
\end{abstract}

\section{Introduction}

The complexity of heart rate variability (HRV) has been well established as an important measure in cardiac health, as it reflects the heart's ability to adapt to sudden perturbations. Multiscale entropy (MSE) [1] is one of the measures assessing the complexity of long-range correlated signals. In HRV studies, MSE curves often have distinguishable profiles under different conditions, such as age and cardiac diseases [2]. QT intervals also exhibit spontaneous beat-to-beat variability, which is useful in monitoring increased risks of fatal ventricular arrhythmias. Only few studies have quantified QT variability (QTV) using MSE [3] and related measures, such as sample entropy [4] and revised MSE [5]. In essence, QT intervals have a MSE profile distinctly different from those of RR intervals [3].

To the best of our knowledge, entropy-based measures have not yet been reported for beat rate dynamics at the cellular level. In this study, we apply the MSE method to quantify the complexity of field potential signals, generated by clusters of human-induced pluripotent stem-cellderived cardiomyocytes (hiPSC-CMs). For this purpose, we extract the interbeat intervals (IBIs) and field potential durations (FPDs) from the field potential. These quantities correspond to the RR and QT intervals of the electrocardiogram (ECG), respectively. The observed effects of age and differentiation methods of hiPSC-CMs on the IBI entropies contribute important information for the characterization of the cell aggregates as an in vitro model of a heart. We demonstrate the ability of the MSE method to distinguish the diseased state of the cell aggregates, which is useful in the study of hereditary cardiac diseases modeled by hiPSC-CMs.

\section{Data and Methods}

\subsection{Electrocardiogram data}

Raw ECG recordings were obtained from the MIT-BIH Normal Sinus Rhythm database of PhysioNet [6]. RR and QT intervals were extracted using the PhysioNet algorithm [6] and other software [7,8]. Low quality signals and ectopic beats were discarded. The final set of the ECG data contains $15 \mathrm{RR}$ and QT interval time series of 24 hours from healthy individuals of 11 women (age from 20 to 45 years) and 4 men (age from 26 to 45 years).

\subsection{Cellular data}

Healthy control hiPSCs were derived from skin fibroblasts of a 55-year-old female and a 44-year-old male. The LQT1-specific hiPSCs were derived from a symptomatic 41-year-old female long QT syndrome (LQTS) patient and an asymptomatic 28-year-old LQT-mutation carrier, both carrying G589D missense mutation in KCNQ1 $[9,10]$. The study was performed under the volunteers' informed consent and approved by the Ethical Committee of Pirkanmaa Hospital District (R08070). The hiPSCs were generated as described in Ref. [11], and cultured and differentiated with the small molecule (SM) [12] and the mouse visceral endoderm-like (END2) cell co-culture method, as previously described in Ref. [10]. The field potential signals of hiPSC-CMs were measured with a six-well multielectrode array. The signals displaying the highest amplitudes, low signal-to-noise ratios, and clear repolarization phases were chosen for the analysis. Two parameters that were extracted were IBIs and FPDs; an IBI is defined as the time 
period between two consecutive depolarization peaks. A FPD is defined as the time period between the depolarization peak and the end of the repolarization peak. The extracted IBI and FPD time series have a length of 10003000 beats.

\subsection{Multiscale entropy analysis}

Entropy-based algorithms measure the degree of regularity to quantify complexity. The multiscale entropy (MSE) method overcomes the limitations of traditional entropy-based algorithms by considering nature of complexity at various scales [2]. The complexity at each scale is quantified by the sample entropy (SampEn) [13], which is obtained from a set of time series that are coarse-grained by scale factors $\tau$. The SampEn values are then plotted over the scale factors to produce a MSE curve. Due to the finite length of real-world data, it is not feasible to assign a single complexity value from the MSE curves, but the curve profiles can be compared between normalized time series.

In this study, we use the same MSE parameters as in the previous studies [2,3], i.e., the epoch length $m=2$ and tolerance level $r=0.15$. For hiPSC-CM aggregates we apply scale factors $1 \leq \tau \leq 10$.

\section{Results and Discussion}

\subsection{ECG vs. cellular level}

For healthy adults, our MSE analysis of RR intervals (from ECG) in Fig. 1(a) is in line with the previous studies. In particular, the result resembles the MSE profile (both the shape and magnitude) of healthy young subjects during sleep [2]. The MSE of the corresponding QT intervals in Fig. 1(a) is significantly smaller than that of RR for scales greater than five $(\mathrm{p}<0.05$ by independent t-test). This reflects the deterministic components of the QT variability (QTV), possibly driven by HRV as a major physiological source of the QTV in the resting condition. A comparable result has been reported in Ref. [3]. The MSE curve of the QT intervals consists of two different regimes. At small scales ( $<5$ in particular), the curve resembles that of the shuffled QT, indicating highly irregular QTV. At larger scales $(>10)$, on the other hand, the profile of the curve is very similar to that of RR. The observation suggests that similar control mechanisms regulating HRV may be assumed for QTV at large scales.

The MSE analysis of IBIs and FPDs for healthy hiPSCCM aggregates is shown in Fig. 1(b). At small scales $(<5)$, the entropy values of IBI and FPD decrease monotonically, forming similar profiles as those of the shuffled series. The white noise-like irregularity may be due to hiPSC-CMs' immaturity and divergent expression levels of atrial, nodal, and ventricular cardiomyocytes [14]. Other factors such as differentiation techniques, age of the cells, and environmental variations may also introduce erratic variability in their beat rates. For scales larger than five beats, the IBI has a constant entropy over the scale, which is a characteristic for the "fractal" $1 / f$ noise. Even though the entropy values are lower than those of the shuffled IBI, we expect an overturn at a larger scale, as long-range correlated IBIs would retain a constant entropy value, while uncorrelated shuffled series would have monotonically decreasing entropy over the scale. On the other hand, the MSE curve of FPDs closely follows that of the shuffled series over the scales up to ten beats. The result agrees well with our previous report [15], in which FPDs of hiPSC-CM aggregates were observed to have scaling exponents comparable to that of white noise at relatively small scales $(<20$ beats $)$.

\section{2. hiPSC-CMs by age and differentiation methods}

The age and differentiation methods of hiPSC-CMs are factors that may affect the cell aggregates' beat rate dynamics. Figure 2 shows the MSE curves for IBI and FPD data, classified by those factors. The difference between the MSE curves is pronounced for IBIs. In particular, the IBI entropies of 26-day-old hiPSC-CM aggregates are significantly larger than the others $(\mathrm{p}<0.05$ for scales $>3$ beats). The observation suggests that the complexity of the IBIs is higher for younger hiPSC-CMs, though no statistically significant difference is found in the entropy values between 47- and 70-day-old hiPSC-CMs.

Two older hiPSC-CM groups are differentiated with the END2 method. Both exhibit monotonically decreasing MSE profiles at short scales, contrary to that of 26-day-old hiPSC-CMs, which are differentiated with the SM method. Therefore, the significant difference in the entropy values may be due to different differentiation methods of the hiPSC-CMs. More systematic investigation is required to determine which factor is more responsible in determining the MSE profile and entropy values.

On the other hand, FPDs show no significant differences between different groups. The MSE profile follows that of the shuffled FPD, as already seen in Fig. 1(b). Therefore, neither age nor differentiation method affects the dynamics of FPDs in the defined scale.

\subsection{Healthy vs. diseased hiPSC-CMs}

The MSE is also evaluated for hiPSC-CMs derived from type-1 long-QT syndrome patients (LQT1-CMs) carrying the KCNQ1 (G589D) mutation. Figure 3 shows three cell groups of (i) healthy wild type (WT) and (ii) symptomatic and (iii) asymptomatic LQT1 cells. Here we use only the IBIs as they contain more interesting information than 

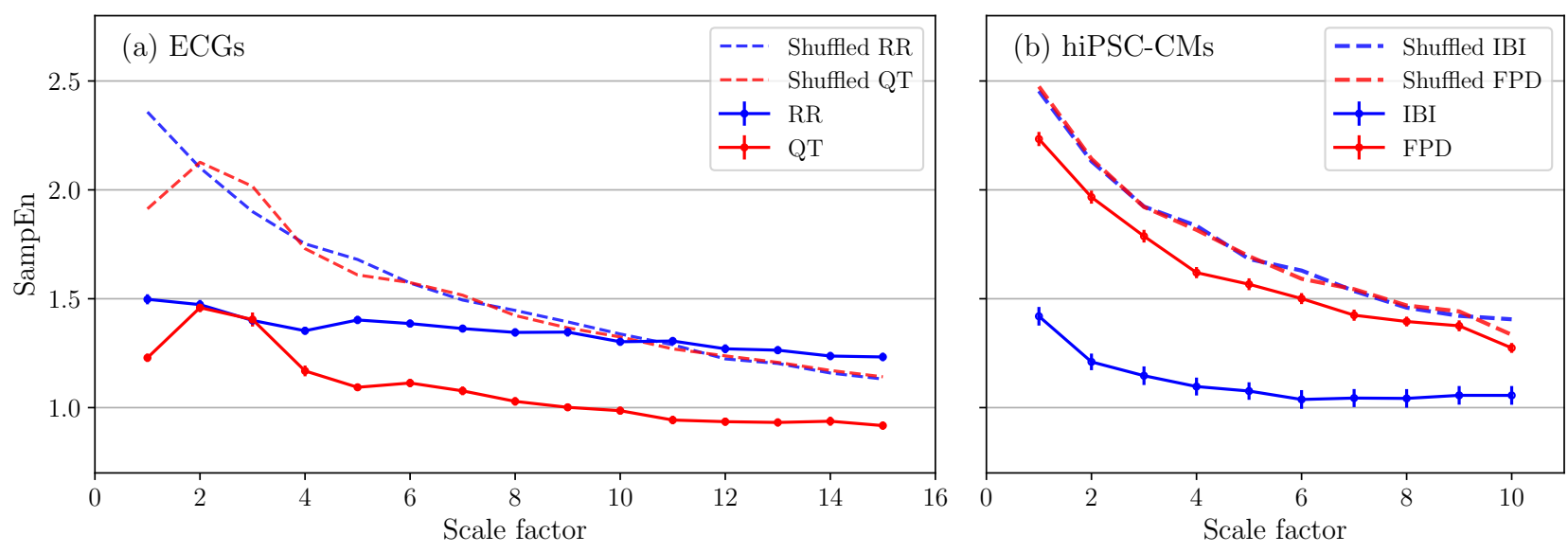

Figure 1. MSE analysis of (a) RR and QT intervals of ECGs and (b) IBIs and FPDs of hiPSC-CM aggregates. MSE analysis of shuffled, i.e., uncorrelated, times series (dashed lines) are included as references.
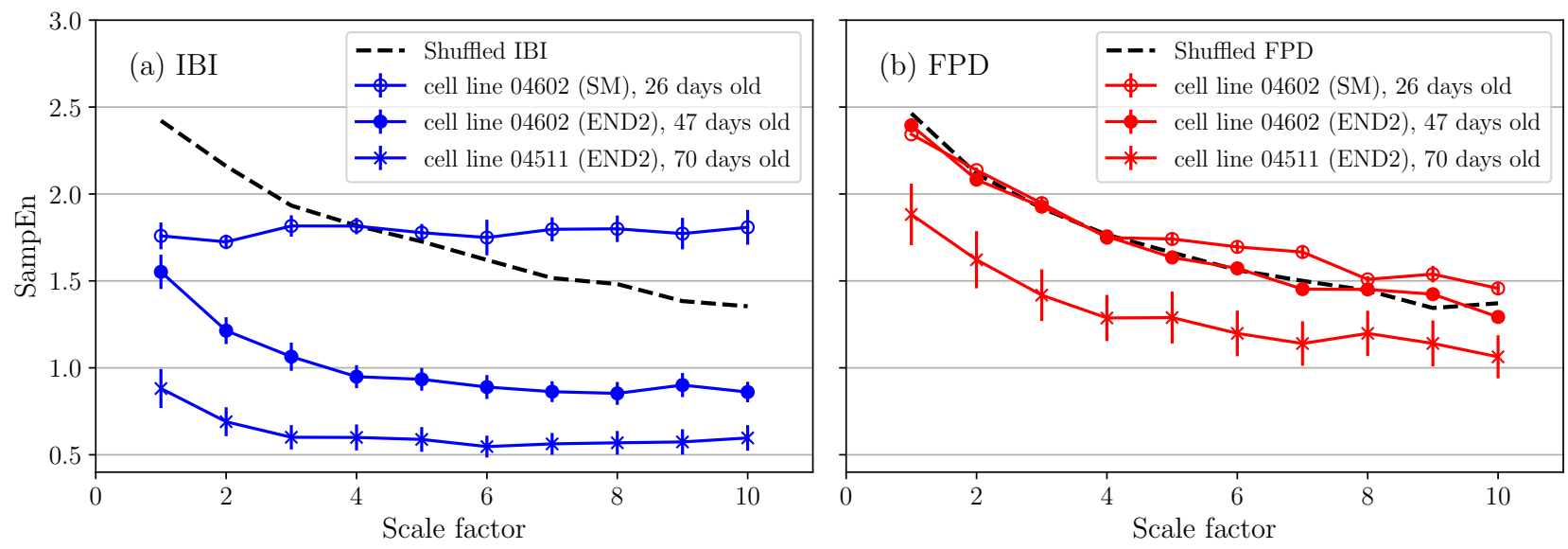

Figure 2. MSE curves of (a) IBI and (b) FPD, classified by their cell lines, differentiation methods, and age of the cells. The MSE curve of shuffled data is added in each plot as a reference. The averages of the curves are shown in Fig. 1 (b).

FPDs in the defined scale regime of fewer than ten beats. The hiPSC-CMs of all three groups are differentiated with the END2 method and have comparable ages ranging 3045 days. Similar to the MSE profiles of 47- and 70-day-old hiPSC-CMs in Fig. 2 (a), all three curves in Fig. 3 share a common feature of monotonically decreasing entropy values over small scales $(<5)$.

For scales larger than five beats, the entropy of symptomatic LQT1-CMs continues to decrease, while those of healthy and asymptomatic LQT1-CMs reach constant values. Thus, the beat rate dynamics of symptomatic LQT1CMs seems to be less complex than that of healthy or asymptomatic LQT1-CMs at scales $5 \ldots 10$ beats. However, the MSE of symptomatic LQT1-CMs still contains a structure that persists over the scale, as it is clearly different from that of uncorrelated noise, represented by the shuffled series (dashed line in Fig. 3). The result indicates that the intrinsic complexity of the hiPSC-CMs can be altered by a diseased state of hiPSC-CMs. It is also in line with our previous study [16], which showed that symptomatic LQT1-CMs exhibit reduced short-term scaling exponents, while healthy and asymptomatic LQT1CMs have scaling exponents close to $1 / f$ noise throughout the scale.

\section{Conclusion}

We have characterized the complexity of beat rates and beat durations of hiPSC-CM aggregates using the MSE method. While RR-equivalent IBIs exhibit scale-invariant entropy values, especially for large scales greater than five consecutive beats, the MSE profiles of QT-equivalent 


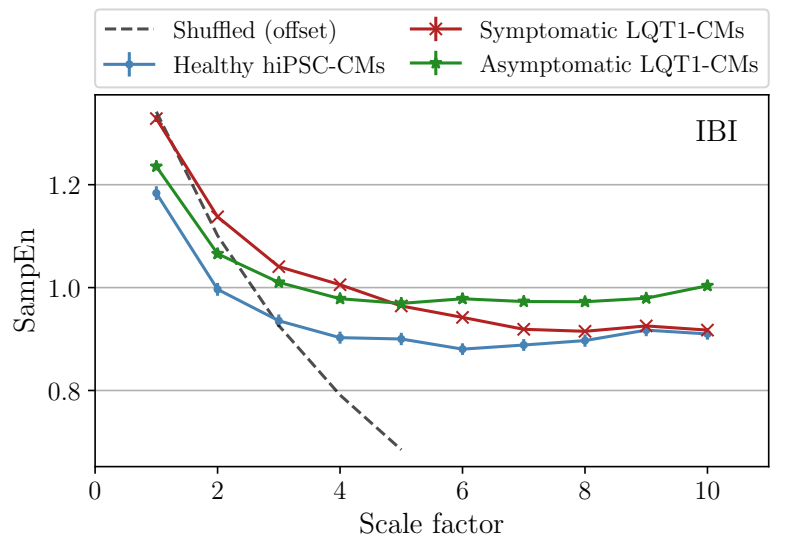

Figure 3. MSE analysis of IBIs of healthy, symptomatic and asymptomatic LQT1-CMs. All hiPSC-CMs are differentiated with the END2 method and aged 30-45 days. The reference MSE of shuffled data (dashed line) is offset to the overlap with other curves for easier comparison.

FPDs follow those of uncorrelated noise. This reflects the irregular nature of FPDs at small scales ( $<10$ beats). We suggest that the age and differentiation method of hiPSCCMs possibly affect the entropy values and profile of IBIs at short scales, though these factors do not affect the FPD results. The MSE analysis of healthy and LQT1 cells also shows that the diseased state of hiPSC-CMs leads to reduced complexity at short scales. The result suggests that the usefulness of the MSE method in studying other hereditary cardiac diseases that can be modeled by hiPSC-CMs.

\section{References}

[1] Costa M, Goldberger AL, Peng CK. Multiscale Entropy Analysis of Complex Physiologic Time Series. Physical Review Letters 2002;89(6):068102.

[2] Costa M, Goldberger AL, Peng CK. Multiscale Entropy Analysis of Biological Signals. Physical Review E 2005; 71(2):021906.

[3] Baumert M, Javorka M, Seeck A, Faber R, Sanders P, Voss A. Multiscale Entropy and Detrended Fluctuation Analysis of QT Interval and Heart Rate Variability During Normal Pregnancy. Computers in Biology and Medicine 2012; 42(3):347-352.

[4] Lewis M, Short A. Sample Entropy of Electrocardiographic RR and QT Time-Series Data During Rest and Exercise. Physiological Measurement 2007;28(6):731.

[5] Bari V, Valencia JF, Vallverdú M, Girardengo G, Marchi A, Bassani T, Caminal P, Cerutti S, George Jr AL, Brink PA, et al. Multiscale Complexity Analysis of the Cardiac Control Identifies Asymptomatic and Symptomatic Patients in Long QT Syndrome Type 1. PLoS One 2014;9(4):e93808.
[6] Goldberger AL, Amaral LA, Glass L, Hausdorff JM, Ivanov PC, Mark RG, Mietus JE, Moody GB, Peng CK, Stanley HE. Physiobank, Physiotoolkit, and Physionet: Components of a New Research Resource for Complex Physiologic Signals. Circulation 2000;101(23):e215-e220.

[7] Silva I, Moody GB. An Open-Source Toolbox for Analysing and Processing Physionet Databases in Matlab and Octave. Journal of Open Research Software 2014;2(1).

[8] Pan J, Tompkins WJ. A Real-Time QRS Detection Algorithm. IEEE Trans Biomed Eng 1985;32(3):230-236.

[9] Kiviaho AL, Ahola A, Larsson K, Penttinen K, Swan H, Pekkanen-Mattila M, Venäläinen $\mathrm{H}$, Paavola K, Hyttinen J, Aalto-Setälä K. Distinct Electrophysiological and Mechanical Beating Phenotypes of Long QT Syndrome Type 1-Specific Cardiomyocytes Carrying Different Mutations. IJC Heart Vasculature 2015;8:19-31.

[10] Kuusela J, Kujala VJ, Kiviaho A, Ojala M, Swan H, Kontula K, Aalto-Setälä K. Effects of Cardioactive Drugs on Human Induced Pluripotent Stem Cell Derived Long QT Syndrome Cardiomyocytes. SpringerPlus 2016;5(1):234.

[11] Takahashi K, Tanabe K, Ohnuki M, Narita M, Ichisaka T, Tomoda K, Yamanaka S. Induction of Pluripotent Stem Cells from Adult Human Fibroblasts by Defined Factors. Cell 2007;131(5):861-872.

[12] Lian X, Zhang J, Azarin SM, Zhu K, Hazeltine LB, Bao X, Hsiao C, Kamp TJ, Palecek SP. Directed Cardiomyocyte Differentiation from Human Pluripotent Stem Cells by Modulating Wnt/ $\beta$-Catenin Signaling Under Fully Defined Conditions. Nature Protocols 2013;8(1):162.

[13] Richman JS, Moorman JR. Physiological Time-Series Analysis Using Approximate Entropy and Sample Entropy. American Journal of Physiology Heart and Circulatory Physiology 2000;278(6):H2039-H2049.

[14] Altomare C, Pianezzi E, Cervio E, Bolis S, Biemmi V, Benzoni P, Camici GG, Moccetti T, Barile L, Vassalli G. Human-Induced Pluripotent Stem Cell-Derived Cardiomyocytes from Cardiac Progenitor Cells: Effects of Selective Ion Channel Blockade. EP Europace 2016; 18(suppl_4):iv67-iv76.

[15] Kim J, Shah D, Potapov I, Latukka J, Aalto-Setälä K, Räsänen E. Scaling and Correlation Properties of RR and QT Intervals at the Cellular Level. Scientific Reports 2018; Submitted.

[16] Kim J, Kuusela J, Aalto-Setälä K, Räsänen E. Shortand Long- Range Correlations in Beat Rate Variability of Human Pluripotent-Stem-Cell-Derived Cardiomyocytes. Computing 2017;44:1.

Address for correspondence:

Jiyeong Kim

Laboratory of Physics, Tampere University of Technology

Korkeakoulunkatu 10, 33720 Tampere

jiyeong.kim@tut.fi 